\title{
Non-Integrability of Some Higher-Order Painlevé Equations in the Sense of Liouville ${ }^{\star}$
}

Ognyan CHRISTOV ${ }^{\dagger}$ and Georgi GEORGIEV ${ }^{\ddagger}$

$\dagger$ Faculty of Mathematics and Informatics, Sofia University, 1164 Sofia, Bulgaria

E-mail: christov@fmi.uni-sofia.bg

$\ddagger$ Department of Mathematics and Informatics, University of Transport, 1574, Sofia, Bulgaria

E-mail: ggeorgiev6@yahoo.com

Received December 10, 2014, in final form June 10, 2015; Published online June 17, 2015

http://dx.doi.org/10.3842/SIGMA.2015.045

Abstract. In this paper we study the equation

$$
w^{(4)}=5 w^{\prime \prime}\left(w^{2}-w^{\prime}\right)+5 w\left(w^{\prime}\right)^{2}-w^{5}+(\lambda z+\alpha) w+\gamma,
$$

which is one of the higher-order Painlevé equations (i.e., equations in the polynomial class having the Painlevé property). Like the classical Painlevé equations, this equation admits a Hamiltonian formulation, Bäcklund transformations and families of rational and special functions. We prove that this equation considered as a Hamiltonian system with parameters $\gamma / \lambda=3 k, \gamma / \lambda=3 k-1, k \in \mathbb{Z}$, is not integrable in Liouville sense by means of rational first integrals. To do that we use the Ziglin-Morales-Ruiz-Ramis approach. Then we study the integrability of the second and third members of the $\mathrm{P}_{\mathrm{II}}$-hierarchy. Again as in the previous case it turns out that the normal variational equations are particular cases of the generalized confluent hypergeometric equations whose differential Galois groups are non-commutative and hence, they are obstructions to integrability.

Key words: Painlevé type equations; Hamiltonian systems; differential Galois groups; generalized confluent hypergeometric equations

2010 Mathematics Subject Classification: 70H05; 70H07; 34M55; 37J30

\section{Introduction}

The Painlevé property for a system of differential equations is the property that its general solution is without movable critical points.

Let us given a nonlinear differential equation

$$
F\left(x, y, y^{\prime}, \ldots, y^{(n)}\right)=0, \quad x, y \in \mathbb{C P}^{1},
$$

where $F$ is a polynomial with respect to $y, y^{\prime}, \ldots, y^{(n)}$. Let $y=\varphi(x)$ be a solution of the above equation, which usually turns out to be a multivalued holomorphic function.

A critical point is a point of ramification of the solution $y=\varphi(x)$. The critical point is called movable if its position depends on the solution $\varphi$, that is, on the constants of integration. For example, the first order equations without movable critical points are the linear equations and the Riccati equations (see [6,31]). Equations with this property are called equations of Painlevé type.

In the beginning of 20th century Painlevé and Gambier investigated this property for secondorder ordinary differential equations. They proved that there are fifty equations possessing the

${ }^{\star}$ This paper is a contribution to the Special Issue on Algebraic Methods in Dynamical Systems. The full collection is available at http://www.emis.de/journals/SIGMA/AMDS2014.html 
Painlevé property. Among them six equations turned out to be new at that time, now called classical Painlevé equations. Although derived in a pure mathematical way, the six Painlevé equations have appeared in many physical applications: in the description of nonlinear waves, in statistical mechanics, in the theory of quantum gravity, in topological field theory, in plasma physics, in the theory of random matrix models and so on.

The classical Painlevé equations have many remarkable properties, in particular they admit a Hamiltonian formulation. In [19] Morales-Ruiz asked the question about the non-integrability as Hamiltonian systems of classical Painlevé equations which have particular rational solutions. This question was answered affirmatively for $\mathrm{P}_{\text {II }}$ with values of the parameter $\alpha=n \in \mathbb{Z}$. For the recent development in the study of the non-integrability of the other Painlevé equations we refer to [28]. In a very recent paper [32] Żoładek and Filipuk have proved that the classical Painlevé equations do not admit a first integral that can be expressed in terms of elementary functions, except for some known cases of $\mathrm{P}_{\mathrm{III}}$ and $\mathrm{P}_{\mathrm{V}}$.

It is natural to extend the question of non-integrability to the higher-order Painlevé equations. Consider the following fourth-order nonlinear ordinary differential equation

$$
w^{(4)}=5 w^{\prime \prime}\left(w^{2}-w^{\prime}\right)+5 w\left(w^{\prime}\right)^{2}-w^{5}+(\lambda z+\alpha) w+\gamma,
$$

where $\lambda, \alpha, \gamma$ are complex parameters.

This equation appears as a group-invariant reduction of the modified Kaup-Kupershmidt (or Sawada-Kotera) equation, see for instance [9, 12]. Then it appears as equation F-XVIII in the classification made by Cosgrove [3] of all fourth- and fifth-order equations with Painlevé property. It is also studied by Gromak [7] from different points of view. It is proven in [12], that (1.1) with $\lambda=1, \alpha=0$ has no polynomial first integral.

Like the classical Painlevé equations, this equation admits a Hamiltonian formulation, Bäcklund transformations and families of rational and special functions. For instance:

- when $\lambda=0, \gamma \neq 0$ it is solved in terms of hyperelliptic functions;

- when $\lambda=0, \gamma=0$ it is solved via elliptic functions;

- when $\gamma=-\lambda / 2, w(z)$ can be expressed in terms of two Painlevé I solutions.

Further, we assume that $\lambda \neq 0$.

The equation (1.1) possesses two families of rational solutions:

I) $\gamma / \lambda=3 k, k \in \mathbb{Z}$

$$
k=0, \quad w=0 ; \quad k=1, \quad w_{(1)}=-\frac{3 \lambda}{\alpha+\lambda z} ; \quad \text { etc. }
$$

II) $\gamma / \lambda=3 k-1, k \in \mathbb{Z}$

$$
k=0, \quad w=\frac{\lambda}{\alpha+\lambda z} ; \quad k=1, \quad w_{(1)}=-\frac{2 \lambda}{\alpha+\lambda z} ; \quad \text { etc. }
$$

In fact, these two families are the only rational solutions of (1.1) [7, Theorem 3].

Denote $q_{1}(z):=w(z), \varepsilon^{2}=1$. Then the equation (1.1) can be presented as two equivalent $2+1 / 2$ degrees of freedom Hamiltonian systems with

$$
H_{\varepsilon}=\frac{1}{2} p_{2}^{2}+\frac{7-9 \varepsilon}{12} q_{2}^{3}+p_{1} q_{2}-\frac{1+3 \varepsilon}{4} p_{1} q_{1}^{2}+\frac{3 \varepsilon-1}{4} q_{2}(\lambda z+\alpha)+\left(\gamma+\frac{3 \varepsilon-1}{4} \lambda\right) q_{1} .
$$

The corresponding system of equations is $\left({ }^{\prime}=d / d z\right)$ :

$$
q_{1}^{\prime}=q_{2}-\frac{3 \varepsilon+1}{4} q_{1}^{2}, \quad p_{1}^{\prime}=\frac{1+3 \varepsilon}{2} p_{1} q_{1}-\gamma-\frac{3 \varepsilon-1}{4} \lambda,
$$




$$
q_{2}^{\prime}=p_{2}, \quad p_{2}^{\prime}=-p_{1}-\frac{7-9 \varepsilon}{4} q_{2}^{2}-\frac{3 \varepsilon-1}{4}(\lambda z+\alpha) .
$$

There exist Bäcklund transformations (see [7]) $T_{1}, T_{2}$ and $T:=T_{2} T_{1}$ for the equation (1.1) acting on the parameters in the following way:

$$
\begin{array}{ll}
T_{1}(\lambda)=\lambda, & T_{1}(\alpha)=\alpha, \quad T_{1}(\gamma)=-\gamma-\lambda, \\
T_{2}(\lambda)=\lambda, \quad T_{2}(\alpha)=\alpha, \quad & T_{2}(\gamma)=-\gamma+2 \lambda, \\
T(\gamma)=\gamma+3 \lambda, \quad T^{-1}(\gamma)=\gamma-3 \lambda .
\end{array}
$$

Gromak has shown that these Bäcklund transformations are birational. It is easy to see that they are canonical also.

We can extend in a natural way the Hamiltonian system (1.2) to a three degrees of freedom autonomous system by denoting $\hat{H}\left(q_{1}, q_{2}, z, p_{1}, p_{2}, F\right):=H_{\varepsilon}+F$. Then we have

$$
\begin{aligned}
\frac{d q_{1}}{d s} & =q_{2}-\frac{3 \varepsilon+1}{4} q_{1}^{2}, & \frac{d p_{1}}{d s} & =\frac{1+3 \varepsilon}{2} p_{1} q_{1}-\gamma-\frac{3 \varepsilon-1}{4} \lambda, \\
\frac{d q_{2}}{d s} & =p_{2}, & \frac{d p_{2}}{d s} & =-p_{1}-\frac{7-9 \varepsilon}{4} q_{2}^{2}-\frac{3 \varepsilon-1}{4}(\lambda z+\alpha), \\
\frac{d z}{d s} & =1, & \frac{d F}{d s} & =-\lambda \frac{3 \varepsilon-1}{4} q_{2} .
\end{aligned}
$$

Our first result is the following

Theorem 1.1. The Hamiltonian system (1.4) with parameters $\gamma / \lambda=3 k, \gamma / \lambda=3 k-1, k \in \mathbb{Z}$ is not integrable in the Liouville sense by means of rational first integrals.

We say that a Hamiltonian system with $n$ degrees of freedom is integrable in the sense of Liouville if there exist $n$ (almost everywhere) independent first integrals in involution.

We use as a tool the Ziglin-Morales-Ruiz-Ramis theorem. We obtain a particular solution of (1.4), write the normal variational equation and study its differential Galois group which appears to be large.

The paper is organized as follows. In Section 2 we summarize the necessary facts about the integrability of Hamiltonian systems and the theory of linear equations with singular points and its relation to the differential Galois theory. In [11] N. Katz and O. Gabber have calculated the Galois groups of some classes of linear equations using purely algebraic arguments - global characterization of semisimple algebras. In Section 3 we apply their result to prove Theorem 1.1. In Section 4 we recover Katz's result about the Galois group for our particular linear equation by giving its topological generators. It turns out that the linear equations which appear here are the confluent generalized hypergeometric equations. We use the approach of Duval and Mitschi [5] for the calculation of the formal monodromy, exponential torus and Stokes matrices in the corresponding case.

In fact, the confluent generalized hypergeometric equations have appeared also along the study of other higher-order Painlevé equations. In Section 5 we prove that the second and the third members of the $\mathrm{P}_{\mathrm{II}}$-hierarchy are non-integrable in the Hamiltonian context for some particular values of the parameters. Again the differential Galois groups of confluent generalized hypergeometric equations are obstructions to integrability. We conjecture that the higher members in the $\mathrm{P}_{\mathrm{II}}$-hierarchy satisfy the same property.

\section{Theory}

In this section we recall some notions and facts about integrability of Hamiltonian systems in the complex domain, the Ziglin-Morales-Ruiz-Ramis theory and their relations with differential Galois groups of linear equations. 
Consider a Hamiltonian system

$$
\dot{x}=X_{H}(x), \quad t \in \mathbb{C}, \quad x \in M
$$

corresponding to an analytic Hamiltonian $H$, defined on the complex $2 n$-dimensional manifold $M$. Suppose the system (2.1) has a non-equilibrium solution $\Psi(t)$. Denote by $\Gamma$ its phase curve. We can write the equation in variation (VE) along this solution

$$
\dot{\xi}=D X_{H}(\Psi(t)) \xi, \quad \xi \in T_{\Gamma} M .
$$

Further, consider the normal bundle of $\Gamma, F:=T_{\Gamma} M / T M$ and let $\pi: T_{\Gamma} M \rightarrow F$ be the natural projection. The equation (2.2) induces an equation on $F$

$$
\dot{\eta}=\pi_{*}\left(D X_{H}(\Psi(t))\left(\pi^{-1} \eta\right), \quad \eta \in F,\right.
$$

which is called the normal variational equation (NVE) along $\Gamma$. The NVE (2.3) admits a first integral $d H$, linear on the fibers of $F$. The level set $F_{p}:=\{\eta \in F \mid d H(\eta)=p\}, p \in \mathbb{C}$, is $(2 n-2)$-dimensional affine bundle over $\Gamma$. We shall call $F_{p}$ the reduced phase space of $(2.3)$ and the restriction of the NVE on $F_{p}$ is called the reduced normal variational equation.

Then the main result of the Ziglin-Morales-Ruiz-Ramis [18] theory is:

Theorem 2.1. Suppose that the Hamiltonian system (2.1) has $n$ meromorphic first integrals in involution. Then the identity component $G^{0}$ of the Galois group of the variational equation is abelian.

Next we consider a linear non-autonomous system

$$
y^{\prime}=A(x) y, \quad y \in \mathbb{C}^{n},
$$

or equivalently a linear homogeneous differential equation

$$
y^{(n)}+a_{1}(x) y^{(n-1)}+\cdots+a_{n}(x) y=0,
$$

with $x \in \mathbb{C P}^{1}$ (which is enough for our purposes) and $A \in \operatorname{gl}(n, \mathbb{C}(x)), a_{j}(x) \in \mathbb{C}(x)$. Let $S:=$ $\left\{x_{1}, \ldots, x_{s}\right\}$ be the set of singular points of (2.4) (or (2.5)) and let $Y(x)$ be a fundamental solution of (2.4) (or (2.5)) at $x_{0} \in \mathbb{C} \backslash S$. By the existence theorem there is a fundamental solution $Y(x)$, analytic in a vicinity of $x_{0}$. The continuation of $Y(x)$ along a nontrivial loop on $\mathbb{C P}^{1}$ defines a linear automorphism of the vector space of all solutions analytic in the neighborhood of $x_{0}$, called the monodromy transformation. Analytically this transformation can be presented in the following way. The linear automorphism $\Delta_{\gamma}$, associated with a loop $\gamma \in \pi_{1}\left(\mathbb{C P}^{1} \backslash S, x_{0}\right)$ corresponds to multiplication of $Y(x)$ from the right by a constant matrix $M_{\gamma}$, called monodromy matrix

$$
\Delta_{\gamma} Y(x)=Y(x) M_{\gamma}
$$

The set of these matrices form the monodromy group. Equivalently, the monodromy group can be defined as a group of automorphisms of the solution space [29, 31].

We may attach another object to the system (2.4) (or (2.5)) - a differential Galois group. A differential field $K$ is a field with a derivation $\partial=$ ', i.e., an additive mapping satisfying Leibnitz rule. A differential automorphism of $K$ is an automorphism commuting with the derivation.

The coefficient field in (2.4) (and (2.5)) is $K=\mathbb{C}(x)$. Let $y_{i j}$ be the elements of the fundamental matrix $Y(x)$. Let $L\left(y_{i j}\right)$ be the extension of $K$ generated by $K$ and $y_{i j}$ - a differential field. This extension is called a Picard-Vessiot extension. Similarly to classical Galois theory 
we define the Galois group $G:=\operatorname{Gal}_{K}(L)=\operatorname{Gal}(L / K)$ to be the group of all differential automorphisms of $L$ leaving the elements of $K$ fixed. The Galois group is, in fact, an algebraic group. It has a unique connected component $G^{0}$ which contains the identity and which is a normal subgroup of finite index. The Galois group $G$ can be represented as an algebraic linear subgroup of $\mathrm{GL}(n, \mathbb{C})$ by

$$
\sigma(Y(x))=Y(x) R_{\sigma},
$$

where $\sigma \in G$ and $R_{\sigma} \in \mathrm{GL}(n, \mathbb{C})$.

We can do the same locally at $a \in \mathbb{C P}^{1}$, replacing $\mathbb{C}(x)$ by the field of germs of meromorphic functions at $a$. In this way we can speak of a local differential Galois group $G_{a}$ of (2.4) at $a \in \mathbb{C P}^{1}$, defined in the same way for Picard-Vessiot extensions of the field $\mathbb{C}\{x-a\}\left[(x-a)^{-1}\right]$.

One should note that by its definition the monodromy group is contained in the differential Galois group of the corresponding system.

We say that two linear systems $y^{\prime}=A(x) y$ and $z^{\prime}=B(x) z$ are $K$-equivalent if the latter is obtained from the first by a $K$-linear change $y=P z, P \in \mathrm{GL}(n, \mathbb{C})$ and $B=P^{-1} A P-P^{-1} P^{\prime}$.

Next, we review some facts from the theory of linear systems with singularities. We call a singular point $x_{i}$ regular if any of the solutions of (2.4) (or of (2.5)) has at most polynomial growth in arbitrary sector with a vertex at $x_{i}$. Otherwise the singular point is called irregular.

We say that the system (2.4) has a singularity of the Fuchs type at $x_{i}$ if $A(x)$ has a simple pole at $x=x_{i}$. For the equation (2.5) the Fuchs type singularity at $x_{i}$ means that the functions $\left(x-x_{i}\right)^{j} a_{j}(x)$ are holomorphic in a neighborhood of $x_{i}$.

If the system (2.4) has a singularity of the Fuchs type, then this singularity is regular. The converse is not true. However, for the equation (2.5) the regular singularities coincide with the singularities of the Fuchs type.

Theorem 2.2 ([26]). For a system with only regular singular points, the differential Galois group coincides with the Zariski closure in $\operatorname{GL}(n, \mathbb{C})$ of the monodromy group.

Now we briefly recall the Ramis description of the local Galois group of (2.4) at 0 which we assume to be an irregular singularity. To the end of the section we follow mainly Mitschi (see [17, pp. 368-370] and [16, pp. 153-159]).

Let $K=\mathbb{C}\{x\}\left[x^{-1}\right]\left(\widehat{K}=\mathbb{C}[[x]]\left[x^{-1}\right]\right)$ be the field of convergent Laurent series near 0 (field of formal Laurent series $), K_{t}=\mathbb{C}\{t\}\left[t^{-1}\right]\left(\widehat{K}=\mathbb{C}[[t]]\left[t^{-1}\right]\right)$ are the same objects with respect to the variable $t$ and $A \in \operatorname{gl}(n, K)$. It is known from the classical theory that there exists a formal fundamental solution to $(2.5)$ :

$$
\widehat{Y}(t)=\widehat{H}(t) x^{L} e^{Q(t)}
$$

where $t^{\sigma}=x, \sigma \in \mathbb{N}^{*}, L=\operatorname{Mat}(n, \mathbb{C}), \widehat{H} \in \operatorname{GL}\left(n, \widehat{K}_{t}\right)$ and $Q=\operatorname{diag}\left(q_{1}, \ldots, q_{n}\right), q_{i} \in t^{-1} \mathbb{C}\left[\frac{1}{t}\right]$, $i=1, \ldots, n$. The integer $\sigma$ is called ramification degree at 0 . Denote also $\zeta=e^{2 \pi i / \sigma}$.

First we recall the formal invariants of (2.5). The change of variable $x \rightarrow x e^{2 \pi i}$ commutes with the derivation, so it defines an element $\widehat{m} \in G$, the formal monodromy ( $t \rightarrow t \zeta$ commutes with the corresponding derivation). Relative to $\widehat{Y}$, the automorphism $\widehat{m}$ can be represented by a matrix $\widehat{M}$ :

$$
\widehat{Y}(t \zeta)=\widehat{Y}(t) \widehat{M}
$$

By definition the exponential torus $\mathcal{T}$ of (2.4) relative to $\widehat{Y}$ is the group of the differential $\widehat{K}_{t}$-automorphisms of the differential extension

$$
\widehat{K}_{t}\left(e^{Q}\right)=\widehat{K}_{t}\left(e^{q_{1}}, e^{q_{2}}, \ldots, e^{q_{n}}\right) \quad \text { of } \quad \widehat{K}_{t} .
$$

$\mathcal{T}$ is isomorphic to $\left(\mathbb{C}^{*}\right)^{l}$, where $l$ is the rank of $\mathbb{Z}$-module generated by the $q_{i}$ 's. 
The matrix $\widehat{M}$, clearly invariant by $\widehat{K}$-equivalence is a formal invariant of $(2.5)$. The same thing applies to the exponential torus $\mathcal{T}$.

Let $V_{d}(\alpha)$ be an open sector in $\mathbb{C}^{*} \backslash\{0\}$ with its vertex at 0 :

$$
V_{d}(\alpha)=\left\{x \in \mathbb{C}^{*}|0<| x \mid<R, d-\frac{\alpha}{2}<\arg (x)<d+\frac{\alpha}{2}\right\}
$$

and let $f$ be a holomorphic function on $V_{d}(\alpha)$. We say that $f$ is asymptotic to $\hat{f}=\sum_{n=0}^{\infty} a_{n} x^{n} \in$ $\mathbb{C}[[x]]$ on $V_{d}(\alpha)$ (in Poincaré sense) if, for every closed subsector $W \subset V_{d}(\alpha)$ there exists a positive constant $M_{W, n}$, such that for every $x \in W$

$$
|x|^{-n}\left|f(x)-\sum_{m=0}^{n-1} a_{m} x^{m}\right| \leq M_{W, n}
$$

for every $n$. We write $f \sim \hat{f}$ on $V_{d}(\alpha)$.

Let us restrict ourselves to the case when all non-zero expressions $\left(q_{i}-q_{j}\right)$ have the same degree, that is, $\left(q_{i}-q_{j}\right)=\left(\lambda_{i}-\lambda_{j}\right) t^{-k}, i, j=1, \ldots, n, k \in \mathbb{N}^{*}$. By the classical theory $[23,27,30]$ we have the following result:

Theorem 2.3. For the system (2.4) with a formal solution (2.6), there exists an actual solution $Y=H x^{L} e^{Q}$, where $H \in \mathrm{GL}(n, \mathbb{C}\{t\})$ has asymptotic expansion $\widehat{H}(H \sim \widehat{H}$ and $Y \sim \widehat{Y})$ in any open angular sector with opening $\pi /(k \sigma)$.

We want to extend the solution $Y$ to sectors with opening greater than $\pi /(k \sigma)$. For this purpose we define:

- a Stokes ray as a direction where, for some $i, j=1, \ldots, n$, one has $\operatorname{Re}\left[q_{i}(t)-q_{j}(t)\right]=0$.

- a singular ray is a direction of maximal decay for some $\exp \left(q_{i}-q_{j}\right)$, i.e., a bisecting ray of a maximal sector where $\operatorname{Re}\left[q_{i}(t)-q_{j}(t)\right]<0$.

Let $d$ be a singular direction for (2.4) at $x=0$, let $d^{+}$and $d^{-}$be nearby directions with arguments $d^{+}=d+\varepsilon, d^{-}=d-\varepsilon$. Then $V^{ \pm}=V_{d^{ \pm}}(\pi /(k \sigma))$ are two overlaping sectors containing $d$. Let $Y^{-}$and $Y^{+}$be actual solutions of (2.4), such that $Y^{-} \sim \widehat{Y}$ in $V^{-}$and $Y^{+} \sim \widehat{Y}$ in $V^{+}$. Hence, we have two actual solutions $Y^{-}, Y^{+}$over $V_{d}(\pi /(k \sigma))$ (by analytic continuation to this sector, $\varepsilon \rightarrow 0)$. Then there exists $S_{d} \in \mathrm{GL}(n, \mathbb{C})$, such that

$$
Y^{-}=Y^{+} S_{d} \text {. }
$$

The matrix $S_{d}$ is called Stokes matrix (or multiplier) with respect to $d$ and $\widehat{Y}$. The Stokes matrices are unipotent. Moreover, they are invariant under $K$-equivalence, that is, they are analytic invariants for (2.4) (see also [1]).

The actual solutions $Y$ are usually obtained by summation procedure of $\widehat{H}$ along non-singular directions in maximal sectors. We will not recall here the summation theory developed by Ramis (see, for instance [23, 29] for more details) because in our particular case there exist fundamental systems of solutions near the irregular point in suitable sectors expressed by Meijer $G$-function [15].

Finally, we have a theorem that generalize the Schlesinger's result for the Fuchsian case.

Theorem 2.4 (Ramis). With respect to the formal solution (2.6) the analytic Galois group of $(2.5)$ at 0 is the Zariski closure in $\mathrm{GL}(n, \mathbb{C})$ of the subgroup generated by the formal monodromy $\widehat{M}$, the exponential torus $\mathcal{T}$ and the Stokes matrices $S_{d}$ for all singular rays.

Now, let $G_{a}$ be the local Galois groups of (2.4), $a \in S$. All $G_{a}$ can be simultaneously identified with closed subgroups of $G$ and the following result holds [17, Proposition 1.3]:

The global Galois group $G$ is topologically generated in $\operatorname{GL}(n, \mathbb{C})$ by the subgroups $G_{a}$, for all $a \in S$. 


\section{Proof of Theorem 1.1}

Consider first the family of rational solutions (I). Take $\gamma / \lambda=0$ or $k=0$ and $w=0$. Then it is straightforward to be seen that

$$
\begin{aligned}
& w=q_{1}=0, \quad q_{2}=0, \quad p_{2}=0, \quad p_{1}=\frac{1-3 \varepsilon}{4}(\lambda s+\alpha), \\
& z=s, \quad F=F_{0}=\text { const }
\end{aligned}
$$

is a particular solution.

Let $\xi_{j}=d q_{j}, \eta_{j}=d p_{j}, j=1,2, \xi_{3}=d s, \eta_{3}=d F$ be the variations. The variational equation along the solution (3.1) takes the form

$$
\begin{array}{ll}
\xi_{1}^{\prime}=\xi_{2}, & \eta_{1}^{\prime}=\frac{1+3 \varepsilon}{2} p_{1} \xi_{1}, \\
\xi_{2}^{\prime}=\eta_{2}, & \eta_{2}^{\prime}=-\eta_{1}+\frac{1-3 \varepsilon}{4} \lambda \xi_{3}, \\
\xi_{3}^{\prime}=0, & \eta_{3}^{\prime}=-\lambda \frac{3 \varepsilon-1}{4} \xi_{2} .
\end{array}
$$

Then the normal variational equation is

$$
\xi_{1}^{\prime}=\xi_{2}, \quad \eta_{1}^{\prime}=\frac{1+3 \varepsilon}{2} p_{1} \xi_{1}, \quad \xi_{2}^{\prime}=\eta_{2}, \quad \eta_{2}^{\prime}=-\eta_{1} .
$$

Reducing (3.2) to a single equation yields

$$
\xi_{1}^{(4)}=(\lambda z+\alpha) \xi_{1}
$$

After setting $z=1 / \tau$ we obtain

$$
y^{(4)}+\frac{12}{\tau} y^{(3)}+\frac{36}{\tau^{2}} y^{(2)}+\frac{24}{\tau^{3}} y^{\prime}-\frac{\lambda+\alpha \tau}{\tau^{9}} y=0
$$

from where $\tau=0$ or $z=\infty$ is an irregular singular point. After changing the independent variable $z \rightarrow \lambda z+\alpha$ we get $(\partial=d / d z)$

$$
L_{1} \xi_{1}=0, \quad L_{1}=\partial^{4}+a z, \quad a:=-1 / \lambda^{4} .
$$

The operator $L_{1}$ is usually called an Airy type operator. It is irreducible and it is obviously invariant under $\partial \rightarrow-\partial$, hence, $L_{1}$ is selfdual in the terminology of [11]. In the same paper Katz [11] has found that the identity component of the Galois group of $L_{1} \xi_{1}=0$ is $G^{0}=\operatorname{Sp}(4, \mathbb{C})$ which is clearly non-commutative. Therefore, by Theorem 2.1 the Hamiltonian system (1.4) is not integrable in a neighborhood of the particular solution (3.1).

Further we consider the second family of rational solutions (II). Take $\gamma / \lambda=-1$ or $k=0$ and $w=\frac{1}{z+\alpha / \lambda}$. Then we have the following particular solution to (1.4)

$$
\begin{aligned}
& w=q_{1}=\frac{1}{s+\frac{\alpha}{\lambda}}, \quad q_{2}=\frac{3}{4} \frac{\varepsilon-1}{\left(s+\frac{\alpha}{\lambda}\right)^{2}}, \quad p_{2}=-\frac{3}{2} \frac{\varepsilon-1}{\left(s+\frac{\alpha}{\lambda}\right)^{3}}, \\
& p_{1}=\frac{1-3 \varepsilon}{4}(\lambda s+\alpha), \quad z=s, \quad F=\frac{3 \lambda}{4} \frac{1-\varepsilon}{s+\frac{\alpha}{\lambda}}+F_{0} .
\end{aligned}
$$

The VE along this solution is

$$
\xi_{1}^{\prime}=\xi_{2}-\frac{1+3 \varepsilon}{2} q_{1} \xi_{1}, \quad \eta_{1}^{\prime}=\frac{1+3 \varepsilon}{2}\left(p_{1} \xi_{1}+q_{1} \eta_{1}\right),
$$




$$
\begin{array}{ll}
\xi_{2}^{\prime}=\eta_{2}, & \eta_{2}^{\prime}=-\eta_{1}+\frac{1-3 \varepsilon}{4} \lambda \xi_{3}+\frac{9 \varepsilon-7}{2} q_{2} \xi_{2}, \\
\xi_{3}^{\prime}=0, & \eta_{3}^{\prime}=-\lambda \frac{3 \varepsilon-1}{4} \xi_{2} .
\end{array}
$$

Then the NVE becomes

$$
\begin{array}{ll}
\xi_{1}^{\prime}=\xi_{2}-\frac{1+3 \varepsilon}{2} q_{1} \xi_{1}, & \eta_{1}^{\prime}=\frac{1+3 \varepsilon}{2}\left(p_{1} \xi_{1}+q_{1} \eta_{1}\right), \\
\xi_{2}^{\prime}=\eta_{2}, & \eta_{2}^{\prime}=-\eta_{1}+\frac{9 \varepsilon-7}{2} q_{2} \xi_{2} .
\end{array}
$$

Again reducing (3.5) to a single equation gives

$$
\xi_{1}^{(4)}-\frac{10}{\left(z+\frac{\alpha}{\lambda}\right)^{2}} \xi_{1}^{\prime \prime}+\frac{20}{\left(z+\frac{\alpha}{\lambda}\right)^{3}} \xi_{1}^{\prime}-\left[\frac{20}{\left(z+\frac{\alpha}{\lambda}\right)^{4}}+\lambda\left(z+\frac{\alpha}{\lambda}\right)\right] \xi_{1}=0
$$

We make some transformations in order to put this equation in appropriate form. First we shift the independent variable $z+\frac{\alpha}{\lambda} \rightarrow z$. Then we make a change $x=\frac{\lambda z^{5}}{5^{4}}$, which is a finite branched covering map $\mathbb{C P}^{1} \rightarrow \mathbb{C P}^{1}$. In general, the differential Galois group is changed under such transformation, but the identity component remains unchanged (see [18, p. 28]). As a result we get

$$
\xi_{1}^{(4)}+\frac{24}{5 x} \xi_{1}^{(3)}+\frac{86}{25 x^{2}} \xi_{1}^{\prime \prime}+\frac{4}{125 x^{3}} \xi_{1}^{\prime}-\left(\frac{4}{125 x^{4}}+\frac{1}{x^{3}}\right) \xi_{1}=0
$$

Finally, we put $u:=x^{2 / 5} \xi_{1}$ from where denoting $\delta=x d / d x$ we obtain

$$
L_{2} u:=\delta\left(\delta-\frac{2}{5}-1\right)\left(\delta+\frac{1}{5}-1\right)\left(\delta+\frac{2}{5}-1\right) u-x u=0 .
$$

The operator $L_{2}$ in (3.6) is a particular case of so-called Kloosterman operators

$$
\mathrm{Kl}=\prod_{1}^{n}\left(\delta-a_{i}\right)+\lambda x
$$

with $n=4, \lambda=-1, a_{1}=0, a_{2}=7 / 5, a_{3}=4 / 5, a_{4}=3 / 5$. Katz has found that (see [11, Theorem 4.5.3, pp. 59-60]) the identity component of the Galois group of $L_{2} u=0$ is $G^{0}=\operatorname{Sp}(4, \mathbb{C})$ which is noncommutative. Hence, by Theorem 2.1 the Hamiltonian system (1.4) is not integrable in a neighborhood of the particular solution (3.4).

To finish the proof note that having the variable $q_{1}=w$ we can obtain the other phase variables from the equations (1.2). Recall that the equation (1.1) has rational solutions only for $\gamma / \lambda=3 k$ and $\gamma / \lambda=3 k-1, k \in \mathbb{Z}$ and therefore, the Hamiltonian system (1.4) has particular rational solutions for these values of the parameters.

For the first family (I) $\gamma / \lambda=3 k, k \in \mathbb{Z}$ we can relate the solution $w=0$ for $\gamma / \lambda=0(k=0)$ and the corresponding rational solution $w_{(k)}$ for $\gamma / \lambda=3 k$ via the Bäcklund transformation $T^{k}$, $k \in \mathbb{Z}$ (1.3). Since these transformations acting on the phase coordinates are birational (and canonical), the non-integrability of the Hamiltonian system (1.4) for $\gamma / \lambda=0(k=0)$ by means of rational first integrals implies the non-integrability of the corresponding Hamiltonian systems for $\gamma / \lambda=3 k, k$ is any integer. Applying the same arguments to the rational solutions of the second family (II), we conclude the non-integrability of the Hamiltonian systems for $\gamma / \lambda=3 k-1$, $k \in \mathbb{Z}$. This ends the proof of Theorem 1.1. 


\section{Stokes matrices}

In this section we explicitly compute the differential Galois group of (3.6) using the approach taken by Duval and Mitschi $[5,16,17]$ based on obtaining the topological generators of the Galois group, namely the formal and analytical invariants of the equation. We focus only on the equation (3.6) because the other equation (3.3) after the change of the independent variable $x=z^{5} /\left(5^{4} \lambda^{4}\right)$ becomes

$$
\delta\left(\delta+\frac{2}{5}-1\right)\left(\delta+\frac{3}{5}-1\right)\left(\delta+\frac{4}{5}-1\right) \xi_{1}-x \xi_{1}=0
$$

which is of similar kind as (3.6).

The following equation

$$
\mathrm{D}_{q p}(y)=\left[(-1)^{q-p} x \prod_{j=1}^{p}\left(\delta+\mu_{j}\right)-\prod_{j=1}^{q}\left(\delta+\nu_{j}-1\right)\right] y=0,
$$

is called generalized confluent hypergeometric equation since it generalizes the classical confluent Kummer equation. Here $\delta=x d / d x, 0 \leq p \leq q, \mu_{j}, \nu_{j} \in \mathbb{C}, \mu_{i}-\mu_{j} \notin \mathbb{Z}$. For this equation the point 0 is a regular singularity and $\infty$ is an irregular singularity, assuming $p<q$. For such kind of equations the local Galois group $G_{0}$ is a subgroup of $G_{\infty}$, so the global Galois group is $G=G_{\infty}$. In what follows we need some notations:

1) $\underline{\alpha}=\left(\alpha_{1}, \ldots, \alpha_{n}\right) \in \mathbb{C}^{n}$.

2) $\langle\underline{\alpha}\rangle_{m}=\prod_{j=1}^{n} \alpha_{j}\left(\alpha_{j}+1\right) \cdots\left(\alpha_{j}+m-1\right)$.

3) For $a \in \mathbb{C}^{p}, b \in\left(\mathbb{C} \backslash \mathbb{Z}^{-}\right)^{q}$, let

$$
{ }_{p} F_{q}(\underline{a} ; \underline{b} \mid x)=\sum_{n \geq 0} \frac{\langle\underline{a}\rangle_{n}}{\langle\underline{b}\rangle_{n}} \frac{x^{n}}{n !}
$$

be the generalized hypergeometric series and

$$
G_{p q}^{m n}\left(x \mid \frac{\underline{a}}{\underline{b}}\right)=\frac{1}{2 \pi i} \int_{C} \frac{\prod_{j=1}^{m} \Gamma\left(b_{j}-s\right) \prod_{j=1}^{n} \Gamma\left(1-a_{j}+s\right)}{\prod_{j=m+1}^{q} \Gamma\left(1-b_{j}+s\right) \prod_{j=n+1}^{q} \Gamma\left(a_{j}-s\right)} x^{s} d s,
$$

be the Meijer $G$-function [15] and $C$ is a suitable path in the complex plane. It is proven that ${ }_{p} F_{q}$ can be expressed as a $G$-function.

4) Let $\widetilde{\mathbb{C}}$ be the Riemann surface of the logarithm, $l_{1}, l_{2} \in \mathbb{R}: l_{1}<l_{2}$. By a sector we understand the following set

$$
\theta\left(l_{1}, l_{2}\right):=\left\{x \in \widetilde{\mathbb{C}} \mid \arg x \in\left(l_{1}, l_{2}\right)\right\}
$$

5) $\sigma=q-p, \zeta=e^{2 \pi i / \sigma}, \lambda=\frac{1}{2}(\sigma+1)+\sum_{j=1}^{p} \mu_{j}-\sum_{j=1}^{q} \nu_{j}$

If $\left(\nu_{j}-\mu_{k}\right) \notin \mathbb{Z}$ for $j=1, \ldots, q$ and $k=1, \ldots, p$, there exists a basis of solutions to (4.1) near $x=0$ given in terms of $G_{p q}^{1 p}$ or ${ }_{p} F_{q-1}$. Similarly, in a neighborhood of $x=\infty$ there 
exists a fundamental system of solutions expressed in terms of $G_{p q}^{q 1}$ and $G_{p q}^{q 0}$ (see [13, 15]). We specialize them for our particular case.

Duval and Mitschi have calculated the formal invariants (formal monodromy and exponential torus) and the analytic invariants (Stokes matrices) for all families of the equations (4.1) assuming $p \geq 1$. Their calculations can be adapted to the case $p=0$ in which we are. It is obvious that the Kloosterman equation is nothing but a $D_{q 0}$ type equation. Note that these equations are generically irreducible over $\mathbb{C}(x)$ (see [2, p. 297] and [5, p. 41] for the proof).

In the rest of the section we carry out detailed calculations in our particular case for the reader's convenience.

Let us rewrite $L_{2}$ from (3.6) in the form

$$
L_{2}=\left(\delta+\nu_{1}-1\right)\left(\delta+\nu_{2}-1\right)\left(\delta+\nu_{3}-1\right)\left(\delta+\nu_{4}-1\right)-x,
$$

which is of type $\mathrm{D}_{40}$ with $\nu_{1}=1, \nu_{2}=-2 / 5, \nu_{3}=1 / 5, \nu_{4}=2 / 5$.

A fundamental system of solutions near $x=0$ of $L_{2} \xi_{1}=0$ is

$$
\begin{aligned}
& \left\{x_{0}^{7 / 5} F_{3}\left(; \frac{8}{5}, \frac{9}{5}, \frac{12}{5} \mid x\right), x_{0}^{3 / 5} F_{3}\left(; \frac{1}{5}, \frac{4}{5}, \frac{8}{5} \mid x\right),{ }_{0} F_{3}\left(;-\frac{2}{5}, \frac{1}{5}, \frac{2}{5} \mid x\right),\right. \\
& \left.x^{4 / 5}{ }_{0} F_{3}\left(; \frac{2}{5}, \frac{6}{5}, \frac{9}{5} \mid x\right)\right\} .
\end{aligned}
$$

Then the monodromy $M_{0}$ around $x=0$ becomes

$$
M_{0}=\operatorname{diag}\left(e^{2 \pi i 2 / 5}, e^{2 \pi i 3 / 5}, 1, e^{2 \pi i 4 / 5}\right) .
$$

Since 0 is regular singular, the monodromy generates the local Galois group $G_{0}$.

Recall that in our case we have $\sigma=4, \zeta=i, \lambda=5 / 2-6 / 5$. We prefer using letters $\zeta$ and $\lambda$ instead of their particular values.

Let us turn to the description of the local Galois group $G_{\infty}$. To define a fundamental system near $x=\infty$ we need one more function. Let $C$ be a path in the complex plane, connecting $-i \infty$ and $i \infty$ and enclosing the points $n-\nu_{j}, j=1, \ldots, 4, n \in \mathbb{N}$. The following function

$$
\mathbb{G}_{0}(x):=G_{04}^{40}\left(\left.x\right|_{\underline{\nu}}\right)=\frac{1}{2 \pi i} \int_{C} \Gamma(1-\underline{\nu}-s) x^{s} d s
$$

is a solution of $L_{2} \xi_{1}=0$, holomorphic in $\theta(-2 \pi, 2 \pi)$. The analytic continuation of $\mathbb{G}_{0}$ in the sector $\theta(-5 \pi, 5 \pi)$ admits the following asymptotic expansion at $x \rightarrow \infty$ (see [5, Propositions 1.2 and 1.3] or [13])

$$
e^{-4 x^{1 / 4}} x^{\lambda / 4} \Theta(x)
$$

where $\Theta$ is a formal series in $x^{-1 / 4}$. It is straightforward that

$$
\mathbb{G}_{0}\left(x e^{-2 \pi i h}\right), \quad h \in \mathbb{Z}
$$

are also solutions of the same equation. In order to get a fundamental solution near $x=\infty$, one needs to pick four of them in our case. Next, we need a particular version of Meijer's expansion theorem which expresses every $G$-function as a finite sum of the functions $\mathbb{G}_{0}\left(x e^{-2 \pi i h}\right)$.

Proposition 4.1 (see [5, Proposition 1.5]). Let $x \in \theta(3 \pi, 5 \pi)$. Then the following identity holds

$$
\mathbb{G}_{0}(x)=A_{1} \mathbb{G}_{0}\left(x e^{-2 \pi i}\right)+A_{2} \mathbb{G}_{0}\left(x e^{-4 \pi i}\right)+B_{0} \mathbb{G}_{0}\left(x e^{-8 \pi i}\right)+B_{1} \mathbb{G}_{0}\left(x e^{-6 \pi i}\right),
$$

where

$$
A_{h}=-\frac{d^{h}}{d x^{h}}\left(\left\langle 1-x e^{-2 \pi i \underline{\nu}}\right\rangle_{1}\right)_{x=0}, \quad B_{h}=e^{2 \pi i \lambda} \frac{d^{h}}{d x^{h}}\left(\left\langle 1-x e^{2 \pi i \underline{\nu}}\right\rangle_{1}\right)_{x=0} .
$$


The formal solutions of $D_{q p}$ at $\infty$ are known $[2,13]$ and can be verified by computer in our particular case. It is more convenient from now on to use a new variable $t=x^{1 / 4}$. Denote by $\underline{L}_{2}$ the equation obtained after the change of variable. Then we have

$$
\begin{array}{ll}
\underline{\Theta}_{-1}(t)=e^{-4 \zeta^{-1} t} t^{\lambda} \underline{\Theta}\left(\zeta^{-1} t\right), & \underline{\Theta}_{0}(t)=e^{-4 \zeta^{0} t} t^{\lambda} \underline{\Theta}\left(\zeta^{0} t\right), \\
\underline{\Theta}_{1}(t)=e^{-4 \zeta^{1} t} t^{\lambda} \underline{\Theta}\left(\zeta^{1} t\right), & \underline{\Theta}_{2}(t)=e^{-4 \zeta^{2} t} t^{\lambda} \underline{\Theta}\left(\zeta^{2} t\right),
\end{array}
$$

where $\underline{\Theta}(t) \in \mathbb{C}\left[\left[t^{-1}\right]\right]$. We denote the basis of formal solutions at $\infty$ of $\underline{L}_{2}$ by

$$
\underline{\Sigma}(t)=\left\{\underline{\Theta}_{-1}(t), \underline{\Theta}_{0}(t), \underline{\Theta}_{1}(t), \underline{\Theta}_{2}(t)\right\} .
$$

In this basis the formal monodromy is $\underline{\Sigma}(\zeta t)=\underline{\Sigma}(t) \widehat{M}_{\infty}$ :

$$
\widehat{M}_{\infty}=e^{2 \pi i \lambda / 4}\left(\begin{array}{cccc}
0 & 0 & 0 & 1 \\
1 & 0 & 0 & 0 \\
0 & 1 & 0 & 0 \\
0 & 0 & 1 & 0
\end{array}\right) .
$$

Since in our case

$$
q_{-1}(t)=-4 \zeta^{-1} t, \quad q_{0}(t)=-4 \zeta^{0} t, \quad q_{1}(t)=-4 \zeta^{1} t, \quad q_{2}(t)=-4 \zeta^{2} t,
$$

the exponential torus is $\mathcal{T} \cong\left(\mathbb{C}^{*}\right)^{2}$ and can be presented by

$$
\mathcal{T}=\left\{\operatorname{diag}\left(t_{1}, t_{2}, t_{1}^{-1}, t_{2}^{-1}\right)\right\}, \quad t_{1}, t_{2} \in \mathbb{C}^{*} .
$$

The Stokes rays can easily calculated from (4.7) to be

$$
\arg t=n \frac{\pi}{4}, \quad n=0, \ldots, 7 .
$$

Similarly the singular rays $d_{s}$, i.e., the rays bisecting the sectors $\operatorname{Re}\left(q_{i}(t)-q_{j}(t)\right)<0$ turn out to be the same as (4.8).

Let us define the sectors

$$
\theta_{n}=\theta\left(-\frac{\pi}{2}+\frac{n-1}{4} \pi, \frac{\pi}{2}+\frac{n}{4} \pi\right), \quad n=0, \ldots, 7 .
$$

The following proposition is proven by Ramis [22] for the general confluent hypergeometric equation $D_{q p}$. We reformulate it for our particular case.

Proposition 4.2. For every sector $\theta_{n}, n \in[0,1,2, \ldots, 7]$, there exists a unique basis of solutions $\Sigma_{n}(t)$ of $\underline{L}_{2}$ in $\theta_{n}$ with asymptotic expansion $\underline{\Sigma}(t)$ at $\infty$.

This solution corresponds to "summation" of $\underline{\Sigma}(t)$ along a direction in the sector $\theta\left(\frac{n-1}{4} \pi, \frac{n}{4} \pi\right)$. As we will see in the sequel we won't need summation because there exist fundamental systems of actual solutions in $\theta_{n}$.

In these notations the Stokes matrix corresponding to the singular ray $\frac{n}{4} \pi, n \in[0,1, \ldots, 7]$ is defined via

$$
\Sigma_{n}(t)=\Sigma_{n+1}(t) S_{n}, \quad t \in \theta_{n} \cap \theta_{n+1} .
$$

Proposition 4.3. Suppose $n$ and $n^{\prime}$ belong to $[0,1, \ldots, 7]$ and $n^{\prime}-n=2$. Then

$$
\Sigma_{n^{\prime}}(\zeta t)=\Sigma_{n}(t) \widehat{M}_{\infty} .
$$


Proof. If $t \in \theta_{n}$, then $\zeta t \in \theta_{n^{\prime}}$ and $\Sigma_{n^{\prime}}(\zeta t) \widehat{M}_{\infty}^{-1}$ is a basis of solutions to $\underline{L}_{2}$ in $\theta_{n}$ which admits the asymptotic expansion $\underline{\Sigma}_{n}(t)$. The uniqueness from Proposition 4.2 gives the desired result.

Proposition 4.4. Let $n \in[0,1, \ldots, 7]$ and $n=2 m+r$. Then

$$
S_{n}=\widehat{M}_{\infty}^{-m} S_{r} \widehat{M}_{\infty}^{m}
$$

Proof. From the relation (4.9) after changing variables we obtain $\Sigma_{n}(\zeta t)=\Sigma_{n+1}(\zeta t) S_{n}$. Proposition 4.3 gives that

$$
\Sigma_{n-2}(t) \widehat{M}_{\infty}=\Sigma_{n+1-2}(t) \widehat{M}_{\infty} S_{n}
$$

This procedure repeated $m$ times yields

$$
\Sigma_{r}(t) \widehat{M}_{\infty}^{m}=\Sigma_{r+1}(t) \widehat{M}_{\infty}^{m} S_{n} .
$$

But by definition we have $\Sigma_{r}(t)=\Sigma_{r+1}(t) S_{r}$ from where the result is immediate.

This proposition reduces the calculation of the Stokes matrices to $S_{0}$ and $S_{1}:=S_{\pi / 4}$ only.

Proposition 4.5. The function $V(t)=\mathbb{G}_{0}\left(t^{4}\right)$ is asymptotic to $\underline{\Theta}_{0}$ in $\theta\left(-\frac{3 \pi}{4}, \frac{5 \pi}{4}\right)$ when $t \rightarrow \infty$.

This is a reformulation of (4.2) and (4.3) in terms of the variable $t$.

Proposition 4.6. If $t \in \theta\left(\frac{3 \pi}{4}, \frac{5 \pi}{4}\right)$, the following identity holds

$$
V(t)-e^{2 \pi i \lambda} V\left(t e^{-2 \pi i}\right)=A_{1} V\left(t \zeta^{-1}\right)+A_{2} V\left(t \zeta^{-2}\right)+B_{1} V\left(t e^{-2 \pi i} \zeta\right) .
$$

This is a version of the formula (4.5) in terms of the variable $t\left(B_{0}=e^{2 \pi i \lambda}, \zeta=e^{2 \pi i / 4}\right)$.

Next, we find fundamental systems of actual solutions near $\infty$ only in $\theta_{0}, \theta_{1}, \theta_{2}$ since we need only $S_{0}$ and $S_{1}$.

Proposition 4.7 (see [5, Proposition 4.8]). Let $n=0,1,2$. The following sets of solutions form fundamental systems of actual solutions in $\theta_{n}$ :

$$
\Sigma_{n}(t):=\left\{Y_{n, j}(t), j \in\{-1,0,1,2\}\right\},
$$

where

$$
\begin{aligned}
& Y_{n,-1}(t)=\zeta^{\lambda} V\left(t \zeta^{-1}\right), \quad n=0,1,2, \\
& Y_{n, 0}(t)=V(t), \quad n=0,1,2, \\
& Y_{n, 1}(t)=\zeta^{-\lambda} V(t \zeta), \quad n=0,1, \\
& Y_{0,2}(t)= \begin{cases}\zeta^{-2 \lambda}\left[V\left(t \zeta^{2}\right)-A_{1} V(t \zeta)\right], \\
\zeta^{-2 \lambda}\left[e^{2 \pi i \lambda} V\left(t e^{-2 \pi i} \zeta^{2}\right)+A_{2} V(t)+B_{1} V\left(t \zeta^{-1}\right)\right], & t \in \theta\left(-\frac{3 \pi}{4}, \frac{\pi}{4}\right),\end{cases} \\
& Y_{1,2}(t)= \begin{cases}\zeta^{-2 \lambda}\left[V\left(t \zeta^{2}\right)-A_{1} V(t \zeta)-A_{2} V(t)\right], \\
\zeta^{-2 \lambda}\left[e^{2 \pi i \lambda} V\left(t e^{-2 \pi i} \zeta^{2}\right)+B_{1} V\left(t \zeta^{-1}\right)\right], & t \in \theta\left(-\frac{\pi}{2}, \frac{\pi}{4}\right),\end{cases} \\
& Y_{2,1}(t)=\left\{\begin{array}{ll}
\zeta^{-\lambda}\left[V(t \zeta)-A_{1} V(t)\right], \\
\zeta^{-\lambda}\left[e^{2 \pi i \lambda} V\left(t e^{-2 \pi i} \zeta\right)+A_{2} V\left(t \zeta^{-1}\right)+B_{1} V\left(t \zeta^{-2}\right)\right], & t \in \theta\left(-\frac{\pi}{4}, \frac{3 \pi}{4}\right),
\end{array},\right. \\
& Y_{2,2}(t)=\left\{\begin{array}{ll}
\zeta^{-2 \lambda}\left[V\left(t \zeta^{2}\right)-A_{1} V(t \zeta)-A_{2} V(t)-B_{1} V\left(t \zeta^{-1}\right)\right], \\
\zeta^{-2 \lambda} e^{2 \pi i \lambda} V\left(t e^{-2 \pi i} \zeta^{2}\right),
\end{array},\right.
\end{aligned}
$$


Proof. Rewriting the formula (4.4) in terms of $t$ gives us solutions of $\underline{L}_{2}$

$$
V\left(t \zeta^{-h}\right), \quad h \in \mathbb{Z}
$$

Using Proposition 4.6 we combine some of them in order to obtain proper asymptotic in $\theta_{n}$. It remains to verify the validity of the formulas (4.11)-(4.14) in the intersection of their definition intervals. We check only (4.11) since the rest are treated in the same way. So, we need to verify that

$$
V\left(t \zeta^{2}\right)-A_{1} V(t \zeta)=e^{2 \pi i \lambda} V\left(t e^{-2 \pi i} \zeta^{2}\right)+A_{2} V(t)+B_{1} V\left(t \zeta^{-1}\right)
$$

is valid in $\theta\left(-\frac{\pi}{4}, \frac{\pi}{4}\right)$. Using (4.10) from Proposition $4.6\left(\zeta^{4}=e^{2 \pi i}\right)$ and making the change $t \rightarrow t \zeta^{2}$ gives the needed result.

Denote by $E_{i j}$ the square matrix with elements 1 at $i, j$ place and zeroes elsewhere.

Proposition 4.8 (see [5, Theorem 5.2]). The Stokes matrices $S_{0}$ and $S_{1}$ are given by the following formulas

$$
S_{0}=\mathbb{I}+\zeta^{-2 \lambda} A_{2} E_{24}, \quad S_{1}=\mathbb{I}+\zeta^{-\lambda} A_{1} E_{23}+e^{-i \pi \lambda} \zeta^{-\lambda} B_{1} E_{14} .
$$

Proof. By definition $\Sigma_{n}(t)=\Sigma_{n+1}(t) S_{n}, t \in \theta_{n} \cap \theta_{n+1}$. For $t \in \theta_{0} \cap \theta_{1}=\theta\left(-\frac{\pi}{2}\right.$, $\left.\frac{\pi}{2}\right)$ from Proposition 4.7 we get

$$
\begin{array}{ll}
Y_{0,-1}(t)=Y_{1,-1}(t), & Y_{0,0}(t)=Y_{1,0}(t), \\
Y_{0,1}(t)=Y_{1,1}(t), & Y_{0,2}(t)=Y_{1,2}(t)+\zeta^{-2 \lambda} A_{2} Y_{1,0}(t) .
\end{array}
$$

Then

$$
\left\{Y_{0,-1}(t), Y_{0,0}(t), Y_{0,1}(t), Y_{0,2}(t)\right\}=\left\{Y_{1,-1}(t), Y_{1,0}(t), Y_{1,1}(t), Y_{1,2}(t)\right\} S_{0}
$$

gives $S_{0}$. Similarly, for $t \in \theta_{1} \cap \theta_{2}=\theta\left(-\frac{\pi}{4}, \frac{3 \pi}{4}\right)$ we have

$$
\begin{array}{ll}
Y_{1,-1}(t)=Y_{2,-1}(t), & Y_{1,0}(t)=Y_{2,0}(t), \\
Y_{1,1}(t)=Y_{2,1}(t)+\zeta^{-\lambda} A_{1} Y_{2,0}, & Y_{1,2}(t)=Y_{2,2}(t)+\zeta^{-2 \lambda} B_{1} \zeta^{-\lambda} Y_{2,-1}(t) .
\end{array}
$$

Then

$$
\left\{Y_{1,-1}(t), Y_{1,0}(t), Y_{1,1}(t), Y_{1,2}(t)\right\}=\left\{Y_{2,-1}(t), Y_{2,0}(t), Y_{2,1}(t), Y_{2,2}(t)\right\} S_{1}
$$

gives $S_{1}$ since $\zeta^{-2 \lambda}=e^{-\pi i \lambda}$.

From the above proposition we know the Stokes matrices $S_{0}$ and $S_{1}$. In our case easy calculations give that

$$
S_{0}=\mathbb{I}+a E_{24}, \quad S_{1}=\mathbb{I}+b E_{23}+c E_{14},
$$

where $a=2 i, c=i \zeta^{-\lambda} e^{2 \pi i / 5}, b=i c$.

Then Proposition 4.4 gives the other Stokes matrices obtained from $S_{0}, S_{1}$ :

$$
\begin{aligned}
& S_{2}=\mathbb{I}+a E_{13}, \quad S_{4}=\mathbb{I}+a E_{42}, \quad S_{6}=\mathbb{I}+a E_{31}, \\
& S_{3}=\mathbb{I}+c E_{12}+i c E_{43}, \quad S_{5}=\mathbb{I}+c E_{41}+i c E_{32}, \quad S_{7}=\mathbb{I}+c E_{34}+i c E_{21} .
\end{aligned}
$$

Now consider the (connected) subgroup topologically generated by the Stokes matrices and the exponential torus $G_{s}=\left\{S_{j}, \mathcal{T}\right\}$ which is normal in the Galois group $G_{\infty}$ [17]. Hence, by 
Theorem 2.4, $G_{\infty}$ is topologically generated by $G_{s}$ and $\widehat{M}_{\infty}$. Let $\mathcal{G}_{s}$ be the Lie algebra of $G_{s}$ $\left(\mathcal{G}_{s} \subset \operatorname{sl}(4, \mathbb{C})\right)$.

To compute $G_{\infty}$ we first determine the Lie algebra $\mathcal{G}_{s}$, then the corresponding connected subgroup $G_{s}$ of $G_{\infty}$ and after that we describe the action of $\widehat{M}_{\infty}$ on $G_{s}$ (see [17]).

We will show that $\mathcal{G}_{s} \cong \operatorname{sp}(4, \mathbb{C})$. Denote $s_{j} \in \mathcal{G}_{s}$ such that $S_{j}=\exp s_{j}$. We have that $\left[s_{2}, s_{6}\right]=a^{2} d_{1}, d_{1}=E_{11}-E_{33}$ and $\left[s_{0}, s_{4}\right]=a^{2} d_{2}, d_{2}=E_{22}-E_{44}$. Then the Lie algebra $\mathcal{G}_{s}$ admits the following basis

$$
\mathcal{B}:=\left\{s_{0}, s_{2}, s_{4}, s_{6}, d_{1}, d_{2}, s_{1}, s_{3}, s_{5}, s_{7}\right\}
$$

Hence, $\mathcal{G}_{s}$ consists of all matrices $V$ such that $V^{T} J_{1}+J_{1} V=0$, where $J_{1}$ is the following skew-symmetric matrix

$$
J_{1}=\left(\begin{array}{cccc}
0 & 0 & i \beta & 0 \\
0 & 0 & 0 & \beta \\
-i \beta & 0 & 0 & 0 \\
0 & -\beta & 0 & 0
\end{array}\right), \quad \beta^{4}=-1 .
$$

Therefore, we get that $G_{s} \cong \operatorname{Sp}(4, \mathbb{C})$ (and $G^{0}=\operatorname{Sp}(4, \mathbb{C})$ ).

Furthermore, $\left(\widehat{M}_{\infty}^{k}\right)^{T} J_{1} \widehat{M}_{\infty}^{k} \neq J_{1}, k=1,2,3,4$, i.e., $\widehat{M}_{\infty}^{k} \notin G_{s}$, but $\widehat{M}_{\infty}^{5}$ already belongs to $G_{s}$. Hence, the formal monodromy generates a finite group $G_{M}$ isomorphic to $\mathbb{Z} / 5 \mathbb{Z}$ acting nontrivially on $G_{s}$. This gives that $G_{\infty}$ (and therefore $G$ ) is isomorphic to a non-trivial semidirect product $\operatorname{Sp}(4, \mathbb{C}) \rtimes \mathbb{Z} / 5 \mathbb{Z}$.

\section{Non-integrability of the second and third members of the $\mathrm{P}_{\mathrm{II}}$-hierarchy}

We have proved that some particular fourth-order Painlevé equation is non-integrable in the Liouville sense for the set of parameters $\gamma / \lambda=3 k$ and $\gamma / \lambda=3 k-1, k \in \mathbb{Z}$. It turns out that the normal variational equations along certain rational solutions are well-known generalized hypergeometric equations whose differential Galois groups can be found. Since generically these groups are large, the non-integrability comes from the Morales-Ruiz-Ramis theorem.

We briefly mention an interesting relation concerning the linear equations that have appeared in this paper. Let $X$ be a smooth complex projective Fano variety. One can define quantum differential equations on $X$ (see, e.g., $[4,8]$ and the references there for details). When the quantum equation is a linear ordinary differential equation Cruz Morales and van der Put [4] confirm Dubrovin's conjecture that the Gram matrix of $X$ coincides with the Stokes matrix of the quantum differential equation (up to certain equivalence). It appears that for $X=\mathbb{P}^{n-1}$ the quantum differential operator is the Airy type operator $\delta^{n}-z$, and for the weighted projective spaces $\mathbb{P}\left(w_{0}, \ldots, w_{n}\right)$ the quantum differential operator is of Kloosterman type or $D_{q 0}$ for certain $q$. The classical Stokes matrices are then computed for these operators using "multisummation" and the "monodromy identity" (see [4] for details).

It is interesting to note that generalized hypergeometric functions and generalized confluent hypergeometric equations are also related with other Painlevé equations. For instance, the classical dilogarithm

$$
\operatorname{Li}_{2}(z)=-\int_{0}^{z} \frac{\ln (1-s)}{s} d s
$$

whose nontrivial monodromy plays an essential role in proving the non-integrability of some Painlevé VI equations studied by Horozov and Stoyanova [10, p. 626] is related to the generalized 
hypergeometric function as

$$
\mathrm{Li}_{2}(z)=z_{3} F_{2}(1,1,1 ; 2,2 \mid z) .
$$

The polylogarithms $\mathrm{Li}_{k}$ have similar representations.

Let us turn our attention to other higher-order Painlevé equations which admit a Hamiltonian formulation. Consider the $\mathrm{P}_{\mathrm{II}}$-hierarchy which is given by (see [14] and the references there)

$$
\mathrm{P}_{\mathrm{II}}^{(n)}:\left(\frac{d}{d z}+2 w\right) \mathcal{L}_{n}\left[w^{\prime}-w^{2}\right]+\sum_{l=1}^{n-1} \beta_{l}\left(\frac{d}{d z}+2 w\right) \mathcal{L}_{l}\left[w^{\prime}-w^{2}\right]=z w+\alpha_{n}, \quad n \geq 1
$$

where $\mathcal{L}_{n}$ is the operator defined by the recursion relation (the Lenard relation)

$$
\frac{d}{d z} \mathcal{L}_{n+1}=\left[\frac{d^{3}}{d z^{3}}+4\left(w^{\prime}-w^{2}\right) \frac{d}{d z}+2\left(w^{\prime}-w^{2}\right)_{z}\right] \mathcal{L}_{n}, \quad \mathcal{L}_{0}\left[w^{\prime}-w^{2}\right]=\frac{1}{2}
$$

and $\beta_{l}$ and $\alpha_{n}$ are arbitrary complex parameters. (Denoting for short $u:=u(z):=w^{\prime}-w^{2}$, one gets consecutively $\mathcal{L}_{1}[u]=u, \mathcal{L}_{2}[u]=u^{\prime \prime}+3 u^{2}, \mathcal{L}_{3}[u]=u^{(4)}+10 u u^{\prime \prime}+5\left(u^{\prime}\right)^{2}+10 u^{3}$ and so on). A particular member of (5.1) is a nonlinear ODE of order $2 n, n \geq 1$. Some authors consider all $\beta_{l}$ to be trivial. The first three members of the $\mathrm{P}_{\mathrm{II}}$-hierarchy are

$$
\begin{aligned}
\mathrm{P}_{\mathrm{II}}^{(1)}: & w^{\prime \prime}-2 w^{3}=z w+\alpha_{1}, \\
\mathrm{P}_{\mathrm{II}}^{(2)}: & w^{(4)}-10 w\left(w w^{\prime \prime}+w^{\prime 2}\right)+6 w^{5}+\beta_{1}\left(w^{\prime \prime}-2 w^{3}\right)=z w+\alpha_{2}, \\
\mathrm{P}_{\mathrm{II}}^{(3)}: & w^{(6)}-14 w^{(4)} w^{2}-56 w^{(3)} w^{\prime} w+70 w^{\prime \prime}\left(w^{4}-w^{\prime 2}\right)+140 w^{3} w^{\prime 2}-42 w\left(w^{\prime \prime}\right)^{2} \\
& -20 w^{7}+\beta_{1}\left[w^{\prime \prime}-2 w^{3}\right]+\beta_{2}\left[w^{(4)}-10 w\left(w w^{\prime \prime}+w^{\prime 2}\right)+6 w^{5}\right]=z w+\alpha_{3} .
\end{aligned}
$$

The equation $\mathrm{P}_{\mathrm{II}}^{(2)}$ appears in [3, p. 58] as F-XVII.

We are interested in the integrability of the Hamiltonian systems corresponding to these equations. The Hamiltonian for $\mathrm{P}_{\mathrm{II}}^{(1)}$ was known long ago from [21] (and also for the other classical Painlevé equations). The Hamiltonian structure for the $\mathrm{P}_{\mathrm{II}}$-hierarchy was found by Mazzocco and Mo [14]. We study the Liouville integrability of the Hamiltonian systems corresponding to the first three members of the $\mathrm{P}_{\mathrm{II}}$-hierarchy, which are "manageable".

Consider first the Hamiltonian for $\mathrm{P}_{\mathrm{II}}^{(1)}$, namely

$$
H^{(1)}=4 p^{2}+\frac{1}{4} q+\frac{1}{4} p q^{2}+2 p z-\frac{1}{2} q \alpha_{1}
$$

where $q=4 w, p=\frac{1}{2}\left(w^{\prime}-w^{2}-\frac{z}{2}\right)$. Extending (5.3) in a natural way to a two degrees of freedom autonomous Hamiltonian system $\hat{H}_{1}=H^{(1)}+F$, one finds $\left({ }^{\prime}=d / d s\right)$ the corresponding equations

$$
q^{\prime}=8 p+\frac{1}{4} q^{2}+2 z, \quad z^{\prime}=1, \quad p^{\prime}=-\frac{1}{4}-\frac{1}{2} p q+\frac{1}{2} \alpha_{1}, \quad F^{\prime}=-2 p .
$$

The system (5.4) admits the following particular solution when $\alpha_{1}=0$

$$
q=0, \quad p=-\frac{1}{4} s, \quad z=s, \quad F=\frac{s^{2}}{4}+F_{0} .
$$

The NVE along (5.5) is

$$
\xi_{1}^{\prime \prime}=z \xi_{1} .
$$


This is the Airy equation whose Galois group is $G=\operatorname{SL}(2, \mathbb{C}) \cong \operatorname{Sp}(2, \mathbb{C})$. The Hamiltonian system (5.4) is therefore non-integrable with rational first integrals, but we know that from [20].

Next we consider the Hamiltonian for $\mathrm{P}_{\mathrm{II}}^{(2)}$ :

$$
H^{(2)}=\frac{q_{2}}{16}+2 z p_{2}-16 p_{1}^{2} p_{2}+16 p_{2}^{2}+\frac{q_{1} q_{2} p_{2}}{8}+\frac{p_{1} p_{2} q_{2}^{2}}{16}+\frac{\alpha_{2}\left(p_{1} q_{2}-q_{1}\right)}{8}+\beta_{1}\left(8 p_{1}-\beta_{1}\right) p_{2},
$$

where $q_{j}, p_{j}, j=1,2$ are expressible via $w$ and its derivatives. Extending as usual to a three degrees of freedom autonomous Hamiltonian system $\hat{H}_{2}=H^{(2)}+F$ we get

$$
\begin{aligned}
q_{1}^{\prime} & =-32 p_{1} p_{2}+\frac{1}{16} p_{2} q_{2}^{2}+\frac{1}{8} q_{2} \alpha_{2}+8 \beta_{1} p_{2}, \\
q_{2}^{\prime} & =2 z-16 p_{1}^{2}+32 p_{2}+\frac{1}{8} q_{1} q_{2}+\frac{1}{16} p_{1} q_{2}^{2}+\beta_{1}\left(8 p_{1}-\beta_{1}\right), \\
p_{1}^{\prime} & =-\frac{1}{8} p_{2} q_{2}+\frac{1}{8} \alpha_{2}, \\
p_{2}^{\prime} & =-\frac{1}{16}-\frac{1}{8} p_{2} q_{1}-\frac{1}{8} p_{1} p_{2} q_{2}-\frac{1}{8} \alpha_{2} p_{1}, \\
z^{\prime} & =1, \quad F^{\prime}=-2 p_{2} .
\end{aligned}
$$

The system (5.6) admits the following particular solution when $\alpha_{2}=0$ :

$$
q_{1}=q_{2}=0, \quad p_{1}=\frac{\beta_{1}}{4}, \quad p_{2}=-\frac{s}{16}, \quad z=s, \quad F=\frac{s^{2}}{16}+F_{0} .
$$

The NVE along the solution (5.7) reduced to a single linear equation is

$$
\xi_{1}^{(4)}-\frac{5}{s} \xi_{1}^{(3)}+\left(\frac{12}{s^{2}}-\frac{\beta_{1} s}{16}\right) \xi_{1}^{\prime \prime}+\left(\frac{\beta_{1}}{16}-\frac{12}{s^{3}}\right) \xi_{1}^{\prime}-\frac{s^{3}}{256} \xi_{1}=0 .
$$

Here we take the case $\beta_{1}=0$ which is simpler. After introducing the new independent variable $z=s^{7} /\left(2^{8} 7^{4}\right)$ the above equation becomes

$$
\delta\left(\delta+\frac{2}{7}-1\right)\left(\delta+\frac{3}{7}-1\right)\left(\delta+\frac{5}{7}-1\right) \xi_{1}-z \xi_{1}=0,
$$

which is an equation of type $D_{40} \xi_{1}=0$ with $\nu_{1}=1, \nu_{2}=2 / 7, \nu_{3}=3 / 7, \nu_{4}=5 / 7$. In a similar way as in Section 4 (or referring to [11]) one obtains that the identity component of the Galois group of $(5.8)$ is $G^{0}=\operatorname{Sp}(4, \mathbb{C})$, which is not commutative. Hence, the Hamiltonian system corresponding to the higher-order Painlevé equation $\mathrm{P}_{\mathrm{II}}^{(2)}$ is not integrable in the Liouville sense.

Finally, let us write the Hamiltonian for $\mathrm{P}_{\text {II }}^{(3)}$

$$
\begin{aligned}
H^{(3)}= & 64 p_{1}^{4}-192 p_{1}^{2} p_{2}+128 p_{1} p_{3}+\frac{1}{64} p_{3} q_{3}^{2}-\frac{1}{64} p_{1} q_{2}^{2}+64 p_{2}^{2}-\frac{1}{32} q_{1} q_{2}+2 z p_{1}+\frac{q_{3}}{64} \\
& -\frac{1}{32} \alpha_{3} q_{3}+8 \beta_{1}\left(p_{1}^{2}-p_{2}\right)+\beta_{2}\left(4 p_{1}^{2} \beta_{2}-4 p_{2} \beta_{2}-32 p_{1}^{3}+64 p_{1} p_{2}-2 p_{1} \beta_{1}\right) .
\end{aligned}
$$

Extending as usual to a four degrees of freedom autonomous Hamiltonian system $\hat{H}_{3}=H^{(3)}+F$, we obtain

$$
\begin{aligned}
& q_{1}^{\prime}=256 p_{1}^{3}-384 p_{1} p_{2}+128 p_{3}-\frac{q_{2}^{2}}{64}+2 z+16 p_{1} \beta_{1}+8 p_{1} \beta_{2}^{2}-96 \beta_{2} p_{1}^{2}+64 p_{2} \beta_{2}-2 \beta_{1} \beta_{2}, \\
& q_{2}^{\prime}=-192 p_{1}^{2}+128 p_{2}-8 \beta_{1}-4 \beta_{2}^{2}+64 p_{1} \beta_{2}, \\
& q_{3}^{\prime}=128 p_{1}+\frac{1}{64} q_{3}^{2},
\end{aligned}
$$




$$
\begin{aligned}
p_{1}^{\prime} & =\frac{1}{32} q_{2}, \\
p_{2}^{\prime} & =\frac{1}{32} p_{1} q_{2}+\frac{1}{32} q_{1}, \\
p_{3}^{\prime} & =-\frac{1}{32} p_{3} q_{3}-\frac{1}{64}+\frac{1}{32} \alpha_{3}, \\
z^{\prime} & =1, \quad F^{\prime}=-2 p_{1} .
\end{aligned}
$$

Here we consider only the case $\beta_{1}=\beta_{2}=0$. When $\alpha_{3}=0$ the system (5.9) admits the following particular solution

$$
q_{1}=q_{2}=q_{3}=0, \quad p_{1}=p_{2}=0, \quad p_{3}=-\frac{s}{64}, \quad z=s, \quad F=F_{0}=\text { const } .
$$

The NVE along the solution (5.10), reduced to a single linear equation becomes

$$
\xi_{1}^{(6)}-\frac{4}{s} \xi_{1}^{(5)}+\frac{12}{s^{2}} \xi_{1}^{(4)}-\frac{24}{s^{3}} \xi_{1}^{(3)}+\frac{24}{s^{4}} \xi_{1}^{\prime \prime}-s \xi_{1}=0 .
$$

After changing the independent variable by $x=s^{7} / 7^{6}$ we obtain

$$
\delta\left(\delta+\frac{1}{7}-1\right)\left(\delta+\frac{2}{7}-1\right)\left(\delta+\frac{3}{7}-1\right)\left(\delta+\frac{4}{7}-1\right)\left(\delta+\frac{6}{7}-1\right) \xi_{1}-x \xi_{1}=0
$$

which is an equation of type $D_{60} \xi_{1}=0$ with $\nu_{1}=1, \nu_{2}=1 / 7, \nu_{3}=2 / 7, \nu_{4}=3 / 7, \nu_{5}=4 / 7$, $\nu_{6}=6 / 7$. We proceed in a similar way as in Section 4. Here

$$
\sigma=q-p=6, \quad \zeta=e^{2 \pi i / 6}, \quad \lambda=\frac{1}{2}-\frac{2}{7} .
$$

The local monodromy at 0 is clear. It is more convenient to use a new variable $t=x^{1 / 6}$. The basis of the formal solutions is straightforward (see for instance $[2,5]$ ) and the formal monodromy is

$$
\widehat{M}_{\infty}=e^{2 \pi i \lambda / 6}\left(\begin{array}{cccccc}
0 & 0 & 0 & 0 & 0 & 1 \\
1 & 0 & 0 & 0 & 0 & 0 \\
0 & 1 & 0 & 0 & 0 & 0 \\
0 & 0 & 1 & 0 & 0 & 0 \\
0 & 0 & 0 & 1 & 0 & 0 \\
0 & 0 & 0 & 0 & 1 & 0
\end{array}\right) .
$$

The exponential torus is again $\mathcal{T} \cong\left(\mathbb{C}^{*}\right)^{2}$

$$
\mathcal{T}=\left\{\operatorname{diag}\left(t_{1}, t_{2}, t_{1}^{-1} t_{2}, t_{1}^{-1}, t_{2}^{-1}, t_{2}^{-1} t_{1}\right)\right\}, \quad t_{1}, t_{2} \in \mathbb{C}^{*} .
$$

The Stokes rays and the singular rays are

$$
\arg t=n \frac{\pi}{6}, \quad n=0, \ldots, 11 .
$$

We define the sectors $\theta_{n}=\theta\left(-\frac{\pi}{2}+\frac{n-1}{6} \pi, \frac{\pi}{2}+\frac{n}{6} \pi\right), n=0, \ldots, 11$. Again we need only $S_{0}$ and $S_{1}:=S_{\pi / 6}$ in order to obtain all Stokes matrices. The Stokes matrices $S_{0}$ and $S_{1}$ are given by (see [5, Theorem 5.2])

$$
S_{0}=\mathbb{I}+a E_{45}+b E_{36}+c E_{21}, \quad S_{1}=\mathbb{I}+d E_{35}+f E_{26},
$$


where $a=\zeta^{-\lambda} A_{1}, b=\zeta^{-3 \lambda} A_{3}, c=\zeta^{\lambda} e^{-2 \pi i \lambda} B_{1}, d=\zeta^{-2 \lambda} A_{2}, f=\zeta^{-\lambda} e^{-\pi i \lambda} B_{2}$. Using (5.11) and (4.6) we get

$$
f=i \zeta^{-\lambda} e^{2 \pi i 2 / 7}, \quad d=f^{2}, \quad c=\frac{i}{f}, \quad b=i, \quad a=i f, \quad f^{3}=-1 .
$$

The matrices $S_{0}$ and $S_{1}$ together with Proposition 4.4 for $n=0, \ldots, 11$ give the rest of the Stokes matrices

$$
\begin{array}{ll}
S_{2}=\mathbb{I}+i f E_{34}+i E_{25}+\frac{i}{f} E_{16}, & S_{3}=\mathbb{I}+f^{2} E_{24}+f E_{15}, \\
S_{4}=\mathbb{I}+i f E_{23}+i E_{14}+\frac{i}{f} E_{65}, & S_{5}=\mathbb{I}+f^{2} E_{13}+f E_{64}, \\
S_{6}=\mathbb{I}+i f E_{12}+i E_{63}+\frac{i}{f} E_{54}, & S_{7}=\mathbb{I}+f^{2} E_{62}+f E_{53}, \\
S_{8}=\mathbb{I}+i f E_{61}+i E_{52}+\frac{i}{f} E_{43}, & S_{9}=\mathbb{I}+f^{2} E_{51}+f E_{42}, \\
S_{10}=\mathbb{I}+i f E_{56}+i E_{41}+\frac{i}{f} E_{32}, & S_{11}=\mathbb{I}+f^{2} E_{46}+f E_{31} .
\end{array}
$$

Now consider again the (connected) subgroup topologically generated by the Stokes matrices and the exponential torus $G_{s}=\left\{S_{j}, \mathcal{T}\right\}$. Let $\mathcal{G}_{s}$ be the Lie algebra of $G_{s}\left(\mathcal{G}_{s} \subset \operatorname{sl}(6, \mathbb{C})\right)$.

Proposition 5.1. $\mathcal{G}_{s} \cong \operatorname{sp}(6, \mathbb{C})$.

Proof. Denote again $s_{j} \in \mathcal{G}_{s}$ such that $S_{j}=\exp s_{j}, j=0, \ldots, 11$ and $\tau_{1}=E_{11}-E_{33}-E_{44}+E_{66}$ and $\tau_{2}=E_{22}+E_{33}-E_{55}-E_{66}$ which belong to Lie $\mathcal{T}$. Direct calculations yield

$$
\begin{aligned}
& {\left[s_{0}, s_{3}\right]=2 i E_{25}, \quad\left[s_{0}, s_{9}\right]=-2 i E_{41}, \quad\left[s_{6}, s_{9}\right]=2 i E_{52},} \\
& {\left[s_{2}, s_{11}\right]=-2 i E_{36}, \quad\left[s_{2}, s_{5}\right]=2 i E_{14}, \quad\left[s_{4}, s_{7}\right]=2 i E_{63},}
\end{aligned}
$$

and hence, $E_{14}, E_{41}, E_{25}, E_{52}, E_{36}, E_{63} \in \mathcal{G}_{s}$.

Additionally we have that the elements

$$
\begin{aligned}
& B_{1}:=E_{11}-E_{44}=\left[s_{8}, s_{2}\right]-\tau_{2}, \quad B_{2}:=E_{22}-E_{55}=\left[s_{9}, s_{3}\right]-B_{1}, \\
& B_{3}:=E_{33}-E_{66}=B_{1}-\tau_{1},
\end{aligned}
$$

and

$$
\begin{aligned}
B_{4} & :=\frac{1}{f} E_{12}-E_{54}=\frac{i}{f^{2}}\left(\left[s_{6}, \tau_{2}\right]-2 i E_{63}\right), \\
B_{5} & :=\frac{1}{f} E_{23}-E_{65}=-i f^{2}\left(\left[\tau_{1}, s_{4}\right]-2 i E_{14}\right), \\
B_{6} & :=f E_{21}-E_{45}=-i f^{2}\left(\left[\tau_{2}, s_{0}\right]-2 i E_{36}\right), \\
B_{7} & :=f E_{32}-E_{56}=-i f^{2}\left(\left[s_{10}, \tau_{1}\right]-2 i E_{41}\right), \\
B_{8} & :=\frac{1}{f^{2}} E_{16}+E_{34}=\frac{i}{f}\left(\left[\tau_{2}, s_{2}\right]-2 i E_{25}\right), \\
B_{9} & :=\frac{1}{f^{2}} E_{61}+E_{43}=-i f\left(\left[s_{8}, \tau_{2}\right]-2 i E_{52}\right)
\end{aligned}
$$

also belong to $\mathcal{G}_{s}$.

Then the Lie algebra $\mathcal{G}_{s}$ admits the following basis :

$$
\mathcal{B}:=\left\{E_{14}, E_{41}, E_{25}, E_{52}, E_{36}, E_{63}, s_{1}, s_{3}, s_{5}, s_{7}, s_{9}, s_{11}, B_{j}, j=1, \ldots, 9\right\} .
$$


Hence, $\mathcal{G}_{s}$ consists of all matrices $V$ such that $V^{T} J_{1}+J_{1} V=0$, where $J_{1}$ is the following skew-symmetric matrix

$$
J_{1}=\left(\begin{array}{cccccc}
0 & 0 & 0 & f^{2} & 0 & 0 \\
0 & 0 & 0 & 0 & f & 0 \\
0 & 0 & 0 & 0 & 0 & 1 \\
-f^{2} & 0 & 0 & 0 & 0 & 0 \\
0 & -f & 0 & 0 & 0 & 0 \\
0 & 0 & -1 & 0 & 0 & 0
\end{array}\right)
$$

from where we get the desired result.

Therefore, we get that $G_{s} \cong \operatorname{Sp}(6, \mathbb{C})\left(\right.$ and $\left.G^{0}=\operatorname{Sp}(6, \mathbb{C})\right)$.

Furthermore, $\left(\widehat{M}_{\infty}^{k}\right)^{T} J_{1} \widehat{M}_{\infty}^{k} \neq J_{1}, k=1, \ldots, 6$, i.e., $\widehat{M}_{\infty}^{k} \notin G_{s}$, but $\widehat{M}_{\infty}^{7}$ already belongs to $G_{s}$. Hence, the formal monodromy generates a finite group $G_{M}$ isomorphic to $\mathbb{Z} / 7 \mathbb{Z}$ acting nontrivially on $G_{s}$. In this way we obtain that $G_{\infty}$ (and therefore $G$ ) is isomorphic to a nontrivial semidirect product $\operatorname{Sp}(6, \mathbb{C}) \rtimes \mathbb{Z} / 7 \mathbb{Z}$.

Hence, the Hamiltonian system corresponding to the higher-order Painlevé equation $\mathrm{P}_{\mathrm{II}}^{(3)}$ is not integrable in the Liouville sense. Summarizing we have the following

Theorem 5.1. Suppose that

(i) $\beta_{1}=\alpha_{2}=0$. Then the Hamiltonian system corresponding to $\mathrm{P}_{\mathrm{II}}^{(2)}$ is not integrable by means of rational $f$ irst integrals;

(ii) $\beta_{1}=\beta_{2}=\alpha_{3}=0$. Then the Hamiltonian system corresponding to $\mathrm{P}_{\mathrm{II}}^{(3)}$ is not integrable by means of rational first integrals.

The study of the other members of the $\mathrm{P}_{\mathrm{II}}$-hierarchy is technically involved. However, we think that the NVE along certain nontrivial solutions reduced to single equations are of the type $D_{q 0} \xi=0$ with $q$ even. Since the identity components of their differential Galois groups are $\operatorname{Sp}(q, \mathbb{C})$, the autonomous Hamiltonian systems corresponding to these equations are nonintegrable.

The result of Theorem 5.1 can be extended to the entire orbits of parameters using Bäcklund transformations and other special solutions recently found by Sakka [24, 25]. This issue will be addressed elsewhere.

\section{Acknowledgements}

The authors are grateful to the referees for their constructive criticism and suggestions. We also would like to thank Ivan Dimitrov for many useful discussions. O.C. acknowledges partial support by Grant 059/2014 with Sofia University.

\section{References}

[1] Balser W., Jurkat W.B., Lutz D.A., Birkhoff invariants and Stokes' multipliers for meromorphic linear differential equations, J. Math. Anal. Appl. 71 (1979), 48-94.

[2] Beukers F., Brownawell W.D., Heckman G., Siegel normality, Ann. of Math. 127 (1988), 279-308.

[3] Cosgrove C.M., Higher-order Painlevé equations in the polynomial class. I. Bureau symbol P2, Stud. Appl. Math. 104 (2000), 1-65.

[4] Cruz Morales J.A., van der Put M., Stokes matrices for the quantum differential equations of some Fano varieties, Eur. J. Math. 1 (2015), 138-153, arXiv:1211.5266. 
[5] Duval A., Mitschi C., Matrices de Stokes et groupe de Galois des équations hypergéométriques confluentes généralisées, Pacific J. Math. 138 (1989), 25-56.

[6] Golubev V.V., Lectures on analytic theory of differential equations, Gos. Izdat., Moscow, 1950 (in Russian).

[7] Gromak V.I., On fourth-order nonlinear differential equations with the Painlevé property, Differ. Equ. 42 (2006), 1076-1085.

[8] Guest M.A., From quantum cohomology to integrable systems, Oxford Graduate Texts in Mathematics, Vol. 15, Oxford University Press, Oxford, 2008.

[9] Hone A.N.W., Non-autonomous Hénon-Heiles systems, Phys. D 118 (1998), 1-16, solv-int/9703005.

[10] Horozov E., Stoyanova T., Non-integrability of some Painlevé VI-equations and dilogarithms, Regul. Chaotic Dyn. 12 (2007), 622-629.

[11] Katz N.M., On the calculation of some differential Galois groups, Invent. Math. 87 (1987), 13-61.

[12] Kudryashov N.A., Transcendents defined by nonlinear fourth-order ordinary differential equations, J. Phys. A: Math. Gen. 32 (1999), 999-1013.

[13] Luke Y.L., The special functions and their approximations, Vol. I, Mathematics in Science and Engineering, Vol. 53, Academic Press, New York - London, 1969.

[14] Mazzocco M., Mo M.Y., The Hamiltonian structure of the second Painlevé hierarchy, Nonlinearity 20 (2007), 2845-2882, nlin.SI/0610066.

[15] Meijer C., On the G-functions. I-VIII, Indag. Math. 8 (1946), 124-134, 213-225, 312-324, 391-400, 468-475, 595-602, 661-670, 713-723.

[16] Mitschi C., Differential Galois groups and $G$-functions, in Differential Equations and Computer Algebra, Comput. Math. Appl., Academic Press, London, 1991, 149-180.

[17] Mitschi C., Differential Galois groups of confluent generalized hypergeometric equations: an approach using Stokes multipliers, Pacific J. Math. 176 (1996), 365-405.

[18] Morales-Ruiz J., Differential Galois theory and non-integrability of Hamiltonian systems, Birkhäuser, Basel, 1989.

[19] Morales-Ruiz J., Kovalevskaya, Liapounov, Painlevé, Ziglin and the differential Galois theory, Regul. Chaotic Dyn. 5 (2000), 251-272.

[20] Morales-Ruiz J., A remark about Painlevé transcendents, Séminaires Congrès 14 (2006), 229-235.

[21] Okamoto K., Studies on the Painlevé equations. III. Second and fourth Painlevé equations, $P_{\mathrm{II}}$ and $P_{\mathrm{IV}}$, Math. Ann. 275 (1986), 221-255.

[22] Ramis J.-P., Filtration Gevrey sur le groupe de Picard Vessiot d'une équation differentielle irrégulière, Informes de Mathematica Serie A-045/85, 1985.

[23] Ramis J.-P., Martinet J., Théorie de Galois différentielle et resommation, in Computer Algebra and Differential Equations, Comput. Math. Appl., Academic Press, London, 1990, 117-214.

[24] Sakka A.H., Bäcklund transformations for first and second Painlevé hierarchies, SIGMA 5 (2009), 024, 11 pages, arXiv:0903.0342.

[25] Sakka A.H., On special solutions of second and fourth Painlevé hierarchies, Phys. Lett. A 373 (2009), 611-615.

[26] Schlesinger L., Handbuch der Theorie der linearen Differentialgleichungen, B.G. Teubner, Leipzig, 1895.

[27] Sibuya Y., Linear differential equations in the complex domain: problems of analytic continuation, Translations of Mathematical Monographs, Vol. 82, Amer. Math. Soc., Providence, RI, 1990.

[28] Stoyanova T., Non-integrability of the fourth Painlevé equation in the Liouville-Arnold sense, Nonlinearity 27 (2014), 1029-1044.

[29] van der Put M., Singer M.F., Galois theory of linear differential equations, Grundlehren der Mathematischen Wissenschaften, Vol. 328, Springer-Verlag, Berlin, 2003.

[30] Wasow W., Asymptotic expansions for ordinary differential equations, Pure and Applied Mathematics, Vol. 14, Interscience Publishers John Wiley \& Sons, Inc., New York - London - Sydney, 1965.

[31] Żołądek H., The monodromy group, Monografie Matematyczne, Vol. 67, Birkhäuser Verlag, Basel, 2006.

[32] Żołądek H., Filipuk G., Painlevé equations, elliptic integrals and elementary functions, J. Differential Equations 258 (2015), 1303-1355. 